\title{
Numerical Solution of Electro-magneto-thermo-mechanical Shock Problem
}

\author{
A. A. El-Bary \\ Department of Basic and Applied Science \\ Arab Academy for Science and Technology \\ P.O. Box 1029 Alexandria, Egypt \\ e-mail: aaelbary@aast.edu
}

(Rec. 20 April 2006)

\begin{abstract}
A conducting half-space, permeated by an initial magnetic field governed by the generalized equations of thermoelasticity is considered. The bounding plane is acted upon by a combination of thermal and mechanical shock. The formulation is applied to both generalizations, Lord-Shulman theory and the Green-Lindsay theory, as well as to the coupled theory. Laplace transform techniques together with the method of potentials are used. The inversion of the Laplace is carried out using a numerical approach. Numerical results for the temperature, the stress and the induced magnetic and electric field distributions are obtained and illustrated graphically for a particular case. Comparisons are made with the results obtained in the case of the absence of the magnetic field.
\end{abstract}

Key words: generalized thermoelasticity, magneto-thermoelasicity, Laplace transforms

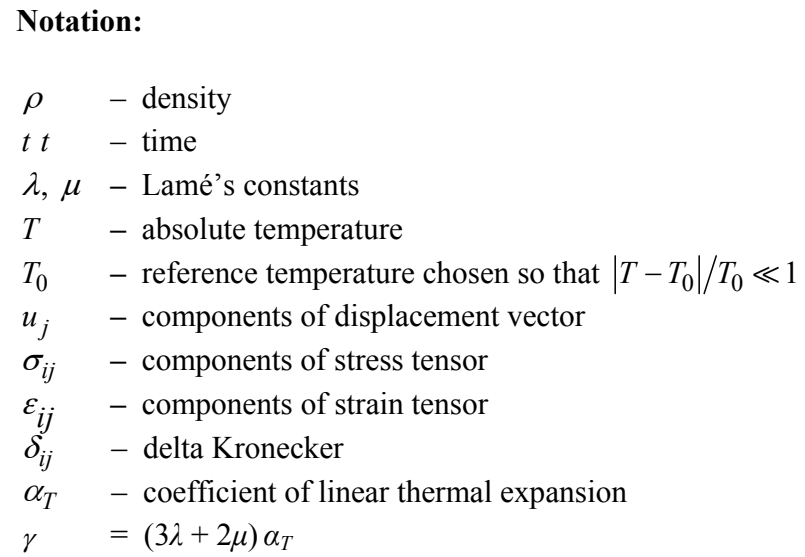

\section{INTRODUCTION}

The classical uncoupled theory of thermoelasticity predicts two phenomena not compatible with physical observations. First, the equation of heat conduction of this theory does not contain any elastic terms; second, the heat equation is of a parabolic type, predicting infinite speeds of propagation for heat waves. Duhamel [1] was the first to consider elastic problems with heat changes. Biot [2] introduced the coupled theory of thermoelasticity to overcome the first shortcoming. The equations of elasticity and of heat conduction for this theory are coupled, eliminating the first paradox of the classical uncoupled theory. However, both theories share the second shortcoming since the heat equation for the coupled theory is also parabolic.

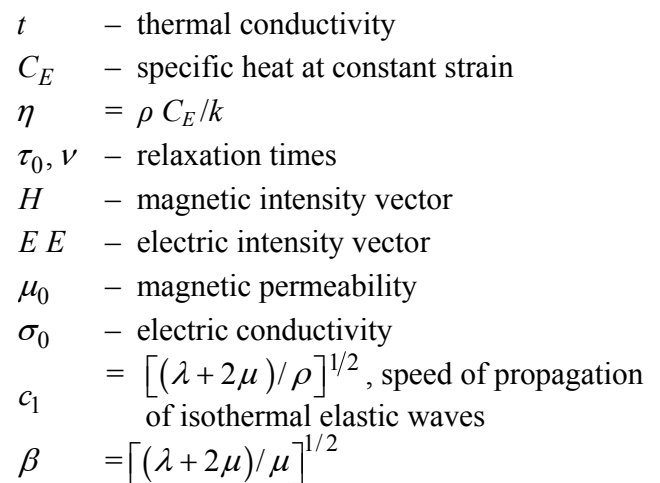

Cattaneo [3] was the first to offer an explicit mathematical correction of the propagation speed defect inherent in Fourier's heat conduction law. Cattaneo's theory allows for the existence of thermal waves, which propagate at finite speeds. Starting from Maxwell's idea [4] and from the paper by Cattaneo [3], an extensive amount of literature [5-10] has contributed to the elimination of the paradox of instantaneous propagation of thermal disturbances. The approach used is known as extended irreversible thermodynamics, which introduces time derivative of the heat flux vector, Cauchy stress tensor and its trace into the classical Fourier law by preserving the entropy principle. Puri and Kythe [9] investigated the effects of using the (Maxwell-Cattaneo) model in Stoke's seStruchtrulem for a viscous fluid. Josef and Preziosi give a detail history of heat con- 
duction theory in $[6,7]$. A history of heat conduction also appears in the review article by Dreyer and Struchtrup [8]. They discuss low temperature heat propagation in dielectric solids where second sound effects are present.

Two generalizations to the coupled thermoelasticity theory were introduced. The first generalization to coupled thermoelasticity is due to Lord and Shulman [11], who introduced the theory of generalized thermoelasticity with one relaxation time (L-S theory). The heat equation of this theory is of the wave-type, it automatically ensures finite speeds of propagation for heat and elastic waves. The remaining governing equations for this theory, namely, the equations of motion and constitutive relations, remain the same as those for the coupled and the uncoupled theories. The second generalization to the coupled theory of elasticity is what is known as the theory of thermoelasticity with two relaxation times or the theory of temperature-ratedependent thermoelasticity. Müller [12], in a review of the thermodynamics of thermoelastic solids, proposed an entropy production inequality, with the help of which he considered restrictions on a class of constitutive equations. A generalization of this inequality was proposed by Green and Laws [13]. Green and Lindsay (G-L theory) obtained an explicit version of the constitutive equations in [14]. These equations were also obtained independently by Şuhubi [15] and Ezzat [16] has obtained the fundamental solution for this theory. This theory contains two constants that act as relaxation times and modify all the equations of the coupled theory, not only the heat equation.

An increasing attention is being devoted to the interaction between magnetic field and strain field in a thrmoelastic solid due to its many applications in the fields of geophysics, plasma physics and related topics. In all papers quoted above it was assumed that the interactions between the two fields take place by means of the Lorentz forces appearing in the equations of motion and by means of a term entering Ohm's law and describing the electric field produced by the velocity of a material particle, moving in a magnetic field. Usually, in these investigations the heat equation under consideration is taken as the uncoupled or the coupled [1] not the generalized one. This attitude is justified in many situations since the solutions obtained using any of these equations differ little quantitatively. However, when short time effects are considered, the fullgeneralized system of equations has to be used a great deal of accuracy is lost.

Among the authors who considered the generalized magneto-thermoelasticity equations are Nayfeh and Nasser [17] who studied the propagation of plane waves in a solid under the influence of an electromagnetic field. Sherief and Ezzat [18] solved a thermal shock half-space problem using asymptotic expansions. Lately, Ezzat [19] studied a problem of half-space when the medium is taken as a perfect conductor.
Sherief [20] studied a thermo-mechanical shock half-space problem, in the absence of magnetic field.

This article is a continuation of the work [20] to include the effect of a transverse magnetic field on an elastic conducting medium.

\section{FORMULATION OF THE PROBLEM}

We shall consider an infinite homogeneous isotropic conducting thermoelastic solid of finite conductivity $\sigma_{0}$ occupying the region $x \geq 0$, where $x$-axis is taken perpendicular to the bounding plane pointing inwards. A constant magnetic field of uniform strength $H$ is permeating the medium in the absence of an external electric field.

The governing equations for generalized thermoelasticity in the presence of a constant magnetic field consist of [18]:

1. The equation of motion

$$
\sigma_{j i, j}+(J \times B)_{i}=\rho \frac{\partial^{2} u_{i}}{\partial t^{2}},
$$

where $B=\mu_{0} H$ is the magnetic induction vector and $j$ is the conduction current density given by Ohm's law as:

$$
J=\sigma_{0}\left[E+\frac{\partial u}{\partial t} \times B\right] .
$$

2. The generalized equation of heat conduction [22]

$$
\begin{gathered}
k T_{, i i}=\rho C_{E}\left(\frac{\partial}{\partial t}+\tau_{0} \frac{\partial^{2}}{\partial t^{2}}\right) T+ \\
+\gamma T_{0}\left(\frac{\partial}{\partial t}+\tau_{0} n_{0} \frac{\partial^{2}}{\partial t^{2}}\right) \varepsilon_{k k}
\end{gathered}
$$

where $n_{0}$ is a constant.

3. The constitutive equation take the form

$$
\begin{gathered}
\sigma_{i j}=\lambda \varepsilon_{k k} \delta_{i j}+2 \mu \varepsilon_{i j}+ \\
-\gamma T_{0}\left(T-T_{0}+v \frac{\partial T}{\partial t}\right) \delta_{i j},
\end{gathered}
$$

where $\varepsilon_{i j}=\frac{1}{2}\left(u_{i, j}+u_{j, i}\right)$.

In the preceding equations, a comma denotes partial differentiation with respect to the space variables $x_{i}$. The summation notation is used.

From the previous equations, we can get the following theories:

1. Coupled theory $\tau_{0}=v=n_{0}=0$

2. Generalized theory with one relaxation time (L-S theory) $v=0, n_{0}=1, \tau_{0}>0$ 
3. Generalized theory with two relaxation times (G-L theory) $n_{0}=0, v \geq \tau_{0}>0$.

Since no external electric field is applied, and the effect of polarization of the ionized medium can be neglected, it follows that the total electric field $E$ vanishes identically inside the medium and the induced electric field produced by the velocity of a material particle, moving in a magnetic field dose not distorts the applied magnetic field. The previous assumption is reasonably true if the magnitude of magnetic viscosity $\left(v_{0}=\sigma_{0} \mu_{0} / \eta\right)$ is very large, which is the case for solids where rather low velocities and electrical conductivity are involved (for instance, Copper, $v_{0}=120.5$, Acrylic, $\left.v_{0}=2.675 \times 10^{24}\right)$. The induced magnetic field which produced in the medium can be ignore if we comparing it with the applied magnetic field. It can be easily seen from the governing equations in [21] that when the electric field vanishes, the coefficient connecting the temperature gradient and electric current as well as coefficient connecting the current density with the heat flow density can be ignored.

For the one-dimensional problems, all the considered functions will depend only on the space variables $x$ and $t$ and the displacement vector has components $(u(x, t), 0,0)$. Let the applied constant magnetic field has the components $\left(0, H_{0}, 0\right)$, then the components of the magnetic induction vector are given by

$$
B_{x}=B_{z}=0, B_{y}=\mu_{0} H_{0}=B_{0} \text { (constant), }
$$

while the components of $F=J \times B$ appearing in Eq. (1) are given by

$$
F_{x}=-\sigma_{0} B_{0}^{2} \frac{\partial u}{\partial t}, F_{y}=F_{z}=0
$$

Using the above values, Eqs. (1-3) reduce to

$$
\begin{gathered}
\rho \frac{\partial^{2} u}{\partial t^{2}}=(\lambda+2 \mu) \frac{\partial^{2} u}{\partial x^{2}}+ \\
-\gamma \frac{\partial}{\partial x}\left(T+v \frac{\partial T}{\partial t}\right)-\sigma_{0} B_{0}^{2} \frac{\partial u}{\partial t} \\
k \frac{\partial^{2} T}{\partial x^{2}}=\rho C_{E}\left(\frac{\partial T}{\partial t}+\tau_{0} \frac{\partial^{2} T}{\partial t^{2}}\right)+ \\
+\gamma T_{0}\left(\frac{\partial^{2} u}{\partial x \partial t}+\tau_{0} n_{0} \frac{\partial^{3} u}{\partial x \partial t^{2}}\right) \\
\sigma=\sigma_{x x}=(\lambda+2 \mu) \frac{\partial u}{\partial x}+ \\
-\gamma T_{0}\left(T-T_{0}+v \frac{\partial T}{\partial t}\right)
\end{gathered}
$$

The governing equations can be put into a more convenient form by using the following non-dimensional variables

$$
\begin{array}{ll}
x^{\prime}=c_{1} \eta x, & u^{\prime}=c_{1} \eta x, \quad t^{\prime}=c_{1}^{2} \eta t,
\end{array}
$$

In terms of these variables, Eqs. (4-6) become (dropping the primes for convenience)

$$
\begin{gathered}
\frac{\partial^{2} u}{\partial t^{2}}=\frac{\partial^{2} u}{\partial x^{2}}-a \frac{\partial}{\partial x}\left(\theta+v \frac{\partial \theta}{\partial t}\right)-M \frac{\partial u}{\partial t} \\
\frac{\partial^{2} \theta}{\partial x^{2}}=\left(\frac{\partial \theta}{\partial t}+\tau_{0} \frac{\partial^{2} \theta}{\partial t^{2}}\right)+ \\
+g\left(\frac{\partial^{2} u}{\partial x \partial t}+\tau_{0} n_{0} \frac{\partial^{3} u}{\partial x \partial t^{2}}\right) \\
\sigma=\sigma_{x x}=\beta^{2} \frac{\partial u}{\partial x}-b\left(\theta+v \frac{\partial \theta}{\partial t}\right)
\end{gathered}
$$

where $b=\frac{\gamma T_{0}}{\mu}, g=\frac{\gamma}{k \eta}$ and $a=\frac{b}{\beta^{2}}$.

The boundary conditions of the problem for $t>0$ are taken as

$$
\begin{gathered}
\sigma(0, t)=f(t), \quad \sigma(\infty, t)=0, \\
\theta(0, t)=g(t), \quad \theta(\infty, t)=0,
\end{gathered}
$$

while the initial conditions are taken to be homogeneous.

\section{SOLUTION \\ IN THE LAPLACE TRANSFORM DOMAIN}

Taking the Laplace transform with parameter s (denoted by a par) of both sides of Eqs. (7-9), we arrive at the following set of equations

$$
\begin{gathered}
\left(\frac{\partial^{2}}{\partial x^{2}}-M s-s^{2}\right) \bar{u}=a(1+v s) \frac{\partial \bar{\theta}}{\partial x} \\
\left(\frac{\partial^{2}}{\partial x^{2}}-s-\tau_{0} s^{2}\right) \bar{\theta}=g s\left(1+\tau_{0} n_{0} s\right) \frac{\partial \bar{u}}{\partial x} \\
\bar{\sigma}=\beta^{2} \frac{\partial \bar{u}}{\partial x}-b(1+v s) \bar{\theta} .
\end{gathered}
$$

Introducing the thermoelastic potential function $\Phi$ defined by the relation 


$$
u=\frac{\partial \Phi}{\partial x} .
$$

Eqs. (12-14) can be written in terms of $\Phi$ as,

$$
\begin{gathered}
\left(D^{2}-M s-s^{2}\right) \bar{\Phi}=a(1+v s) \bar{\theta}, \\
\left(D^{2}-s-\tau_{0} s^{2}\right) \bar{\theta}=g s\left(1+\tau_{0} n_{0} s\right) D^{2} \bar{\Phi}, \\
\bar{\sigma}=\beta^{2} D^{2} \bar{\Phi}-b(1+v s) \bar{\theta},
\end{gathered}
$$

where $D$ denotes $\frac{\partial}{\partial x}$.

The boundary conditions, expressed in terms of $\Phi$, take the form

$$
\begin{gathered}
\bar{\Phi}(0, s)=\frac{\bar{f}(s)}{\beta^{2} s(M+s)}, \\
\beta^{2} D^{2} \bar{\Phi}(0, s)=\bar{f}(s)+b(1+v s) \bar{g}(s), \\
\bar{\Phi}(\infty, s)=D \bar{\Phi}(\infty, s)=D^{2} \bar{\Phi}(\infty, s)=0 .
\end{gathered}
$$

Eliminating $\bar{\theta}$ between (16) and (17), we obtain the following fourth order differential equation satisfied by $\bar{\Phi}$

$$
\begin{gathered}
\left\{D^{4}-D^{2}\left[s^{2}+M s+s\left(1+\tau_{0} s\right)+\varepsilon s(1+v s)\left(1+n_{0} \tau_{0} s\right)\right]+\right. \\
\left.+s^{2}(M+s)\left(1+\tau_{0} s\right) \bar{\Phi}\right\}=0,
\end{gathered}
$$

where $\varepsilon=b g$.

The solution of Eq. (22) satisfying the boundary conditions (21) at infinity may be expressed as

$$
\Phi(x, s)=A_{1}(s) e^{-k_{1} x}+A_{2}(s) e^{-k_{2} x},
$$

where $A_{1}(s)$ and $A_{2}(s)$ are some parameters depending on $s$ only and $k_{1}, k_{2}$ are the roots with positive real parts of the characteristic Eq. (22), given by

$$
\begin{gathered}
k_{1,2}^{2}=\frac{s}{2}\left[s^{2}+M s+s\left(1+\tau_{0} s\right)+\varepsilon s(1+v s)\left(1+n_{0} \tau_{0} s\right) \pm\right. \\
\pm\left(\left[s^{2}+M s+s\left(1+\tau_{0} s\right)+\varepsilon s(1+v s)\left(1+n_{0} \tau_{0} s\right)\right]^{2}+\right. \\
\left.\left.-4 s^{2}(M+s)\left(1+\tau_{0} s\right) s\right)^{\frac{1}{2}}\right] .
\end{gathered}
$$

Using Eqs. (20) and (21), we obtain two equations in the unknown parameters $A_{1}$ and $A_{2}$ whose solution give

$$
\begin{gathered}
A_{1}=\frac{1}{\beta^{2} s(s+M)\left(k_{1}^{2}-k_{2}^{2}\right)} \times \\
\times\left\{s b(s+M)(1+v s) \bar{g}(s)+\left[s(s+M)-k_{2}^{2}\right] \bar{f}(s)\right\}, \\
A_{2}=\frac{1}{\beta^{2} s(s+M)\left(k_{1}^{2}-k_{2}^{2}\right)} \times \\
\times\left\{\left[k_{1}^{2}-s(s+M)\right] \bar{f}(s)-s b(s+M)(1+v s) \bar{g}(s)\right\} .
\end{gathered}
$$

Substituting the above values into Eq. (23), we obtain

$$
\bar{\Phi}(x, s)=\bar{\Phi}_{1}(x, s)+\bar{\Phi}_{2}(x, s),
$$

where

$$
\begin{gathered}
\bar{\Phi}_{1}(x, s)=\frac{b(1+v s) \bar{g}(s)}{\beta^{2}\left(k_{1}^{2}-k_{2}^{2}\right)}\left[e^{-k_{1} x}-e^{-k_{2} x}\right], \\
\bar{\Phi}_{2}(x, s)=\frac{-\bar{f}(s)}{s(s+M) \beta^{2}\left(k_{1}^{2}-k_{2}^{2}\right)} \times \\
\times\left\{\left[k_{2}^{2}-s(s+M)\right] e^{-k_{1} x}-\left[k_{1}^{2}-s(s+M)\right] e^{-k_{2} x}\right\} .
\end{gathered}
$$

The function $\bar{\Phi}_{1}(x, s)$ represents the response of the system to the thermal shock problem while $\bar{\Phi}_{2}(x, s)$ represents the response to the mechanical shock.

Substituting from Eqs. (25-27) into Eq. (16), we obtain

$$
\bar{\theta}(x, s)=\bar{\theta}_{1}(x, s)+\bar{\theta}_{2}(x, s),
$$

where

$$
\begin{gathered}
\bar{\theta}_{1}(x, s)=\frac{\bar{g}(s)}{k_{1}^{2}-k_{2}^{2}} \times \\
\times\left\{\left[k_{1}^{2}-s(s+M)\right] e^{-k_{1} x}-\left[k_{2}^{2}-s(s+M)\right] e^{-k_{2} x}\right\}, \\
\bar{\theta}_{2}(x, s)=\frac{-\left[k_{1}^{2}-s(s+M)\right]\left[k_{2}^{2}-s(s+M)\right] \bar{f}(s)}{b s(s+M)(1+v s)\left(k_{1}^{2}-k_{2}^{2}\right)} \times \\
\times\left[e^{-k_{1} x}-e^{-k_{2} x}\right] .
\end{gathered}
$$

Using the following relation between the sum and product of the roots of the characteristic Eq. (22), namely

$$
\begin{aligned}
k_{1}^{2}+ & k_{2}^{2}=s^{2}+M s+s\left(1+\tau_{0} s\right)+ \\
+ & \varepsilon s(1+v s)\left(1+n_{0} \tau_{0} s\right),
\end{aligned}
$$




$$
k_{1}^{2} k_{2}^{2}=s^{2}(M+s)\left(1+\tau_{0} s\right) .
$$

The expression for $\bar{\theta}_{2}$ can be written in a simplified form as

$$
\bar{\theta}_{2}(x, s)=\frac{-\varepsilon(s+M) \bar{f}(s)}{b(1+v s)\left(k_{1}^{2}-k_{2}^{2}\right)}\left[e^{-k_{1} x}-e^{-k_{2} x}\right] .
$$

Substituting from Eqs. (25-27) into Eq. (18), we obtain

$$
\begin{gathered}
\bar{\sigma}(x, s)=\bar{\sigma}_{1}(x, s)+\bar{\sigma}_{2}(x, s), \\
\bar{\sigma}_{1}=\frac{b s(s+M)(1+v s) \bar{g}(s)}{k_{1}^{2}-k_{2}^{2}}\left[e^{-k_{1} x}-e^{-k_{2} x}\right], \\
\bar{\sigma}_{2}(x, s)=\frac{-\bar{f}(s)}{k_{1}^{2}-k_{2}^{2}} \times \\
\times\left\{\left[k_{1}^{2}-s(s+M)\right] e^{-k_{1} x}-\left[k_{2}^{2}-s(s+M)\right] e^{-k_{2} x}\right\} .
\end{gathered}
$$

Eqs. (25)-(34) give the complete solution of the problem in the Laplace domain.

As an example to illustrate the applicability of the previous results, we shall consider the case of a thermomechanical shock in the form of pulse given by

$$
g(t)=\theta_{0} \xi(t) \text { and } f(t)=\sigma_{0} \xi(t),
$$

where the pulse function $\xi(t)$ is defined as

$$
\xi(t)=H(t)-H(t-l)=\left\{\begin{array}{lc}
1 & \text { if } 0 \leq t \leq l \\
0 & \text { otherwise }
\end{array} .\right.
$$

The Laplace transform for Eq. (35) is given by

$$
\bar{g}(s)=\frac{\theta_{0}\left(1-e^{-s l}\right)}{s} \quad \text { and } \quad \bar{f}(s)=\frac{\sigma_{0}\left(1-e^{-s l}\right)}{s} \text {. }
$$

\section{INVERSION}

\section{OF THE LAPLACE TRANSFORMS}

In order to invert the Laplace transform in the above equations, we adopt a numerical inversion method based on a Fourier series expansion [23]. In this method, the inverse $g_{0}(t)$ of the Laplace transform $\bar{g}_{0}(s)$ is approximated by the relation

$$
\begin{gathered}
g_{0}(t)=\frac{e^{c t}}{t_{1}} \times \\
\times\left[\frac{1}{2} \bar{g}_{0}(c)+\operatorname{Re}\left(\sum_{k=1}^{\infty} e^{i k \pi t / t_{1}} \bar{g}\left(c+i k \pi / t_{1}\right)\right)\right], \\
0 \leq t \leq 2 t_{1},
\end{gathered}
$$

where $N$ is a sufficiently large integer representing the number of terms in the truncated infinite Fourier series. $N$ must chosen such that

$$
e^{c t} \operatorname{Re}\left[e^{i N \pi t / t_{1}} \bar{g}_{0}\left(c+i N \pi / t_{1}\right)\right] \leq \varepsilon_{1},
$$

where $\varepsilon_{1}$ is a persecuted small positive number that corresponds to the degree of accuracy to be achieved. The parameter $c$ is a positive free parameter that must be greater than the real parts of all singularities of $\bar{g}_{0}(s)$. The optimal choice of $c$ was obtained according to the criteria described in [23].

In order to find the temperature distribution $\theta$, we use expression (34) with $\theta$ and $\bar{\theta}$ replacing $g_{0}$ and $\bar{g}_{0}(s)$, respectively. This procedure is repeated for the functions of the stress and induced magnetic and electric field distribution.

\section{NUMERICAL RESULTS}

The copper material was chosen for purposes of numerical evaluations. The constants of the problem are shown in Table 1.The values taken for the shock parameters are $\theta_{0}=\sigma_{0}=1$. The computations were carried out for three values of time, namely $t=0.05, t=0.1$ and $t=0.15$ to show the time evolution of the temperature and the induced magnetic and electric field distributions. A comparison is made for stress distribution with the results obtained in the absence of a magnetic field. The all results are shown in Figs. 1-12.

The graph of the temperature $\theta_{1}$ due to the thermal shock only is shown in Fig. 1, while that of the temperature $\theta_{2}$ due to mechanical shock only is shown in Fig. 2.

Table 1. Values of the constants

\begin{tabular}{llll}
\hline$k=386$ & $\alpha_{T}=1.78(10)^{-5}$ & $C_{E}=383.1$ & $\eta=8886.73$ \\
$\mu_{0}=3.86(10)^{10}$ & $\lambda=7.76(10)^{10}$ & $\beta^{2}=4$ & $\rho=8954$ \\
$\tau_{0}=0.02$ & $v=0.02$ & $B_{0}=0.042$ & $g=1.61$ \\
$c_{1}=4.158(10)^{3}$ & $\mu_{0}=4 \pi(10)^{-7}$ & $V=1.39(10)^{-5}$ & $T_{0}=293$ \\
$\varepsilon=0.0168$ & $l=0.15$ & & \\
\hline
\end{tabular}

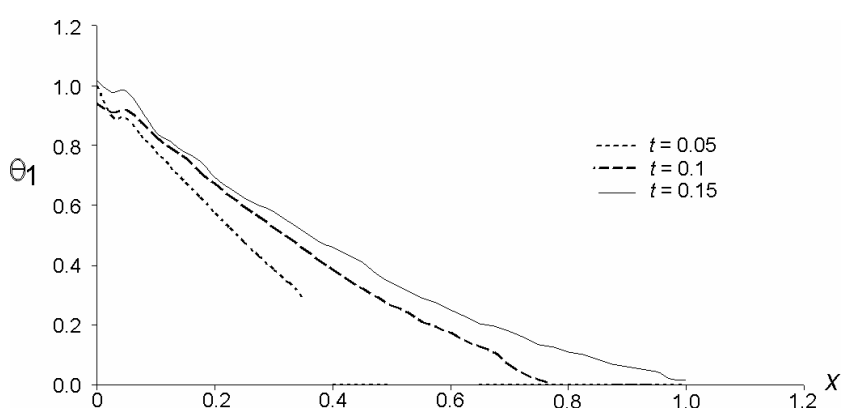

Fig. 1. Temperature distribution due to thermal shock 


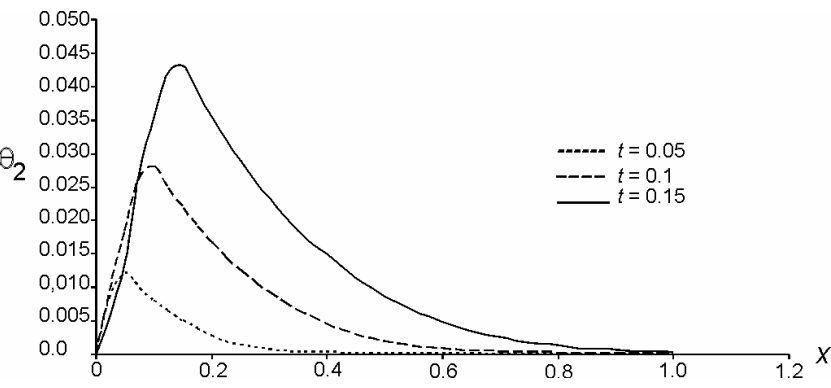

Fig. 2. Temperature distribution due to mechanical shock

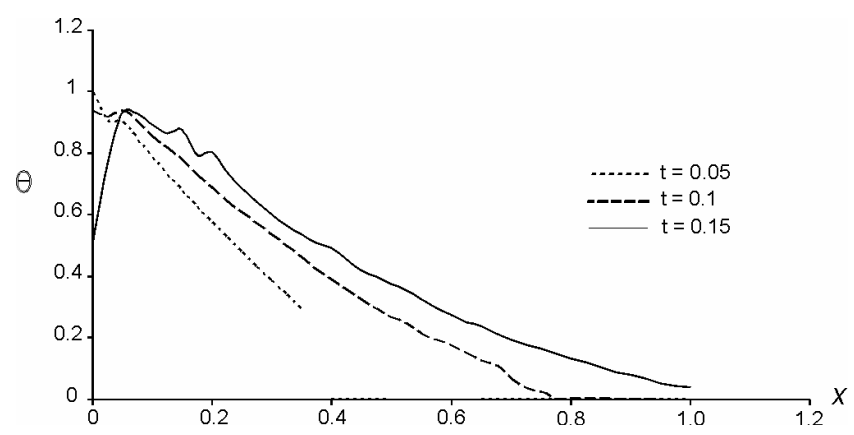

Fig. 3. Total temperature distribution

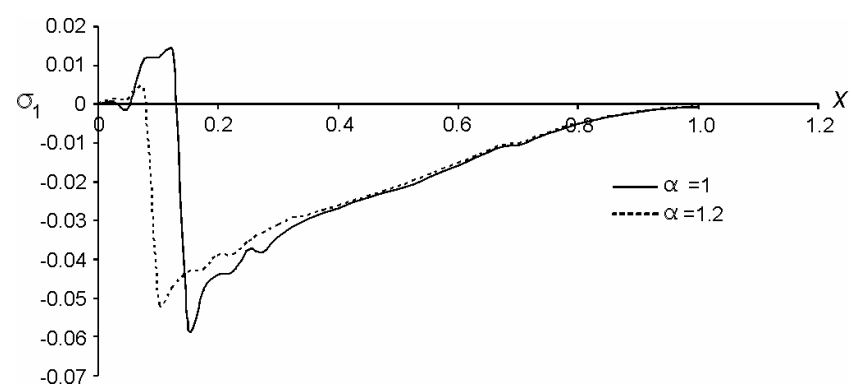

Fig. 4. Stress distribution due to thermal shock in perfect conductng medium

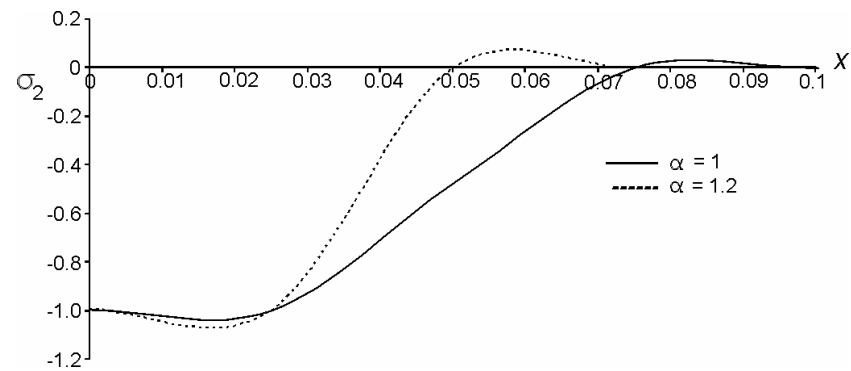

Fig. 5. Stress distribution due to thermal shock in perfect conductng medium

The total temperature distribution is shown in Fig. 3. As expected the values those of $\theta_{2}$. Similarly, the stresses $\sigma_{1}$, $\sigma_{2}$ and $\sigma$ are shown in Figs. 4-6, the induced magnetic field distributions $h_{1}, h_{2}$ and $h$ are shown in Figs. 7-9 and the induced electric field distributions are shown in Figs. 10-12. It was found that the stress $\sigma_{2}$ dominates $\sigma_{1}$, the induced magnetic field $h_{2}$ dominates $h_{1}$ and the induced electric field $E_{2}$ dominates $E_{1}$. The function $\theta_{2}$ was found to be

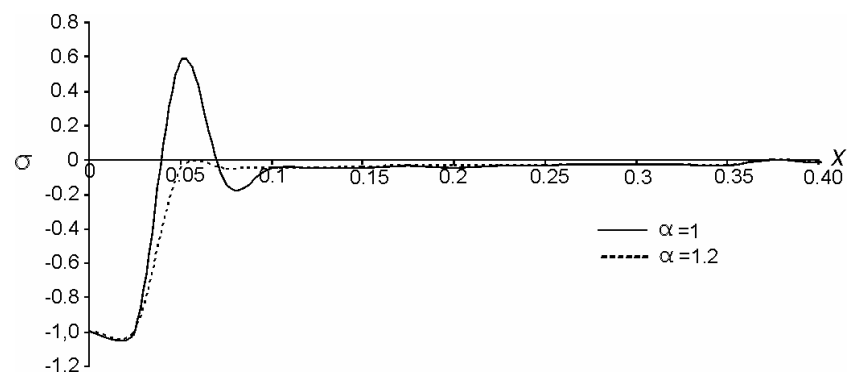

Fig. 6. Total stress distribution in perfect conducting medium

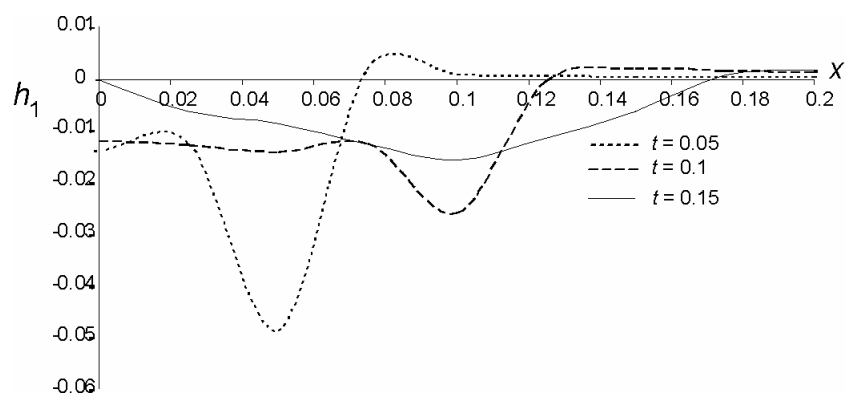

Fig. 7. Induced magnetic field due to thermal shock

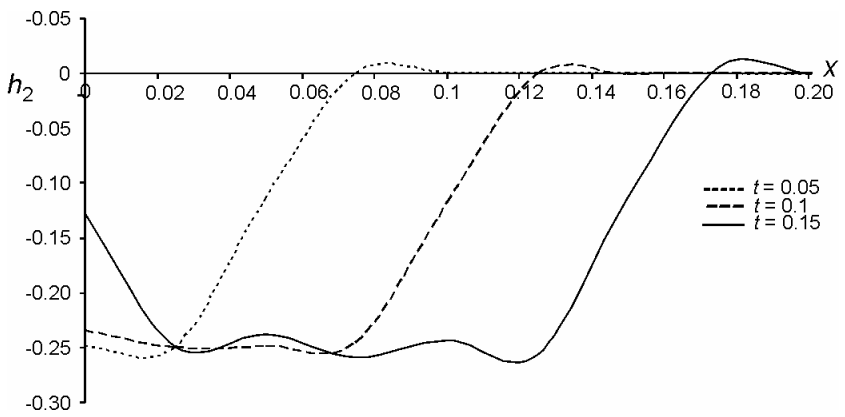

Fig. 8. Induced magnetic field due to thermal shock

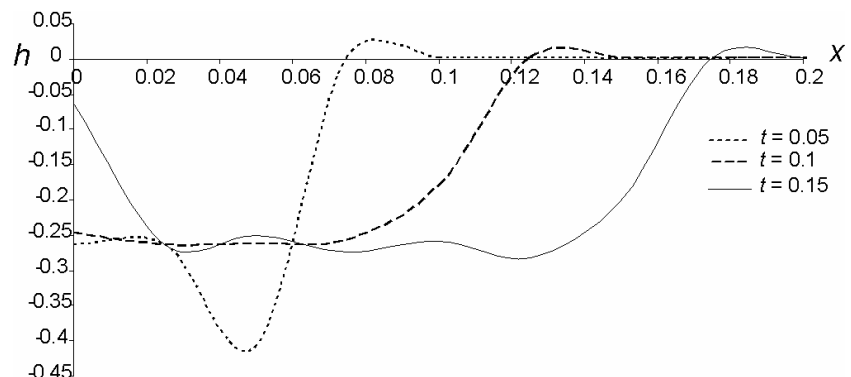

Fig. 9. Total induced magnetic field in perfect conducting medium 


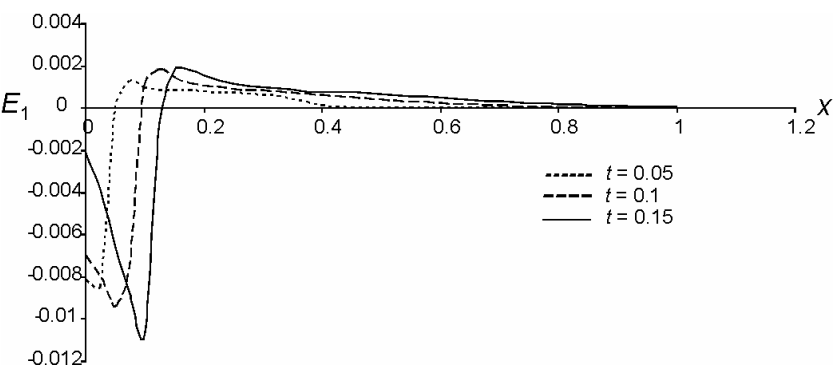

Fig. 10. Induced electric field due to thermal shock

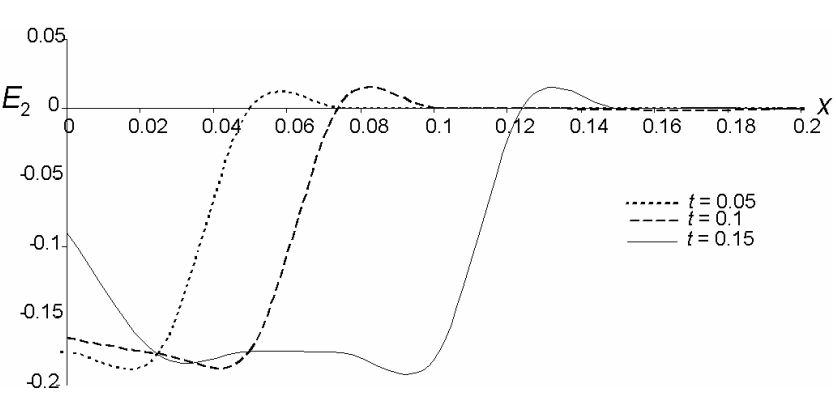

Fig. 11. Induced electric field due to thermal shock

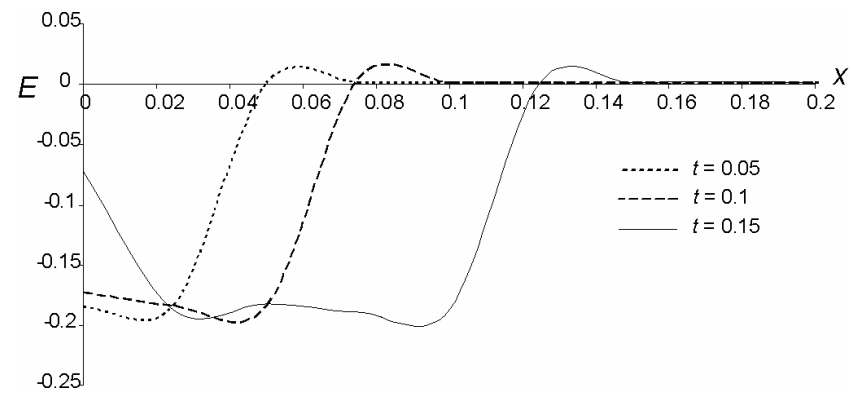

Fig. 12. Total induced electric field in perfect conducting medium

continuous function for all values of $x$ and $t$. For $t \leq 1$, the other functions, namely $\theta_{1}, \sigma_{1}, \sigma_{2}, h_{1}, h_{2}, E_{1}$ and $E_{2}$ all have finite jumps at different positions. It clear that the location of these jumps increase linearly with the increase of the time $t$ while their magnitudes decrease exponentially with the increase of time $t$.

The phenomenon of finite speeds of propagation is manifested in all these figures. For smallest values of time considered we see that the effect of a thermal-mechanical shock on perfect conducting medium localized in a region adjacent to the bounding plane. This region expands with the passage of time to fill the half-space for the largest value of time. This is not the case when the coupled equation of heat conduction is used.
The presence of the magnetic field which acts to the conducting elastic medium raises the velocity of the dilatational elastic waves from $c_{1}$ to $c_{0}=\left(c_{1}^{2}+\mu_{0} H_{0}^{2} / \rho\right)^{1 / 2}$, the modified electromagnetic elastic wave is propagated with velocity $c_{0}$, and that is, with the same velocity as the modified elastic wave that produces a jump in stress. This field shifts the stress discontinuity, discovered at the point $x=c_{1} t$ under purely thermoelastic conditions [24], to the point $x=\sqrt{1+M} \quad c_{1} t$ : it also affects the magnitude of the jump at the discontinuity as seen from Figs. 4-6.

\section{References}

[1] J. H. Duhamel, Second memoir, sur les phenomenes thermomechanique, J. de L' Ecole Polytechnique 15 (1837).

[2] M. Biot, Thermoelasticity and irreversible thermodynamics, J. Appl. Phys. 27 (1956).

[3] C. Cattaneo, Sullacondizione del calore, Atti. Sem. Mat. Fis. Univ. Modena 3 (1948).

[4] C. Truesdell and R. G. Muncaster, Fundamental of Maxwell's kinetic theory of a simple monatomic gas, Acad. Press, New York (1980).

[5] D. E. Glass and B. Vick, Hyperbolic heat conduction with surface radiation, Int. J. Heat Mass Transfer 28, 1823 (1985).

[6] D. D. Joseph and L. Preziosi, Heat waves, Rev. Modern Phys. 61, 41 (1989).

[7] D. D. Joseph and L. Preziosi, Addendum to the paper: heat waves, Rev. Modern Phys. 62, 375 (1989).

[8] W. Dreyer and H. Struchtrup, Heat pulse experiments revisited, Count. Mech. Thermodyn. 5, 3 (1993).

[9] P. Puri and P. K. Kythe, Non-classical thermal effects in Stoke's second problem, Acta Mech. 112, 1 (1995).

[10] D. S. Chandrasekharaiah, Hyperbolic thermoelasticity. A review of recent literature, Appl. Mech. Rev. 51, 705 (1998).

[11] H. Lord and Y. Shulman, A generalized dynamical theory of thermoelasticity, Mech. Phys. Solid. 15, 299 (1967).

[12] I. Müller, The coldness, a universal function in thermoelastic solids, Arch. Rat. Mech. Anal. 41, 319 (1971).

[13] A. Green and N. Laws, On the entropy production inequality, Arch. Rat. Anal. 54, 7 (1972).

[14] A. Green and K. Lindsay, Thermoelasticity, J. Elast. 2, 7 (1972).

[15] E. Şuhubi, Thermoelastic solids, in: A. C. Eringen (ED), Cont. Phys. II, Academic Press, New York (1975) Ch. 2.

[16] M. Ezzat, Fundamental solution in thermoelasticity with two relaxation times for cylindrical regions, Int. J. Engng. Sci. 33, 2011 (1995).

[17] A. Nayfeh and S. Nemat-Nasser, Electromagneto-Thermoelastic Plane Waves in Solids with Thermal Relaxation, J. Appl. Mech. Series E 39, 108 (1972).

[18] H. Sherief and M. Ezzat, A thermal-shock problem in magneto-thermoelasticity with thermal relaxation, Int. J. Solids and Structures 33, 4449 (1996).

[19] M. Ezzat, Generation of generalized magneto-thermoelasticity waves by thermal shock in a perfectly conducting halfspace, J. Thermal Stresses 20, 617 (1997).

[20] H. Sherief, A thermal-mechanical shock problem for thermoelasticity with two relaxation time, Int. J. Engng. Sci. 32, 313 (1994). 
[21] M. Ezzat and A. El-Karamany, Magnetothermoelasticity with two relaxation times in conducting medium with variable electrical and thermal conductivity, J. App. Math and Computaions 142, 449 (2003).

[22] M. Ezzat and A. El-Karamani, The uniqueness and reciprocity theorems for generalized thermoviscoelasticity for anisotropic media, J. Thermal Stresses 25, 507 (2002).
[23] G. Honig and Hirdes, A Method for the Numerical Inversion of the Laplace Transform, J. Comp. Appl. Math. 10, 113(1984).

[24] B. A. Boley and J. H. Weiner, Theory of thermal stresses, Wiley, New York, 1960. 